\title{
Infrared recognition technology and its development trend in the middle segment of ballistic missile
}

\author{
Hua-yu Dai, Yan-li Xu and Shuang zhao \\ The academy of equipment, Beijing 101416, China \\ 948791290@qq.com
}

Keywords: Infrared target recognition, Missile defense system, Infrared warning, temperature variation

\begin{abstract}
Aiming at the features that different kinds of threat target groups have different infrared characteristics in the middle flight of ballistic missile,the characteristics of mid-course of ballistic missile and infrared target recognition technology are studied.Based on temperature, infrared imaging, radiation intensity the three typical parameters of infrared characteristics, the recognition mechanism and the infrared photoelectric method used in the anti-missile target recognition system are discussed in this paper.Finally, take the United States as an example, describes the current development status and main problems of ballistic missile infrared target recognition technology, and on this basis,discussed the direction of priority development of infrared detection in our country.
\end{abstract}

\section{Introduction}

Under the conditions of modern warfare with information technology as the core.Ballistic missiles with its long range,power,high accuracy and great penetration ability and other advantages,since the advent, it has become the most important weapon that in the hands of owner.It is an extremely important deterrent.Meanwhile,manufacturing technology of ballistic missile penetration and a variety of technology has gradually matured,at present, there are more than 40 countries and regions in the world with ballistic missiles. In the periphery of China,some 10 countries already have the ability to produce ballistic missiles.Therefore,to accelerate the research of ballistic missile defense technology is particularly important.

Target recognition as the core technology of ballistic missile defense system $^{[1]}$, has become the focus of the major military powers in the field of research.At the present stage, the main identification methods include two kinds of radar recognition and infrared recognition.Compared with radar identification technology,Space-based infrared recognition with large detection range,the ground clutter affect little,and the advantages of passive detection,it will play an important role in the future ballistic missile defense.

The main work of this paper is to analyze some characteristics of the middle part of the missile.Then, characteristics of the infrared identification of intermediate ballistic missile target are summarized ,and the current domestic and foreign target recognition methods and the latest development of ballistic missiles.Summed up the general advice about ballistic midcourse target recognition

\section{Characteristics analysis of middle segment of ballistic missile}

Ballistic missile in flight process mainly experienced three stages: boosting phase, mid-course, terminal phase (reentry). From the end of the boosting phase to the middle of the reentry phase, the missile flight will go through the following process: booster stage rocket is turned off, the warhead and the missile body are separated, the target group including the warhead , the debris and the decoy is generated, finally reentry atmosphere. When the ballistic missile is flying in the mid-course, the warhead and decoy constitute the target group, and the target characteristic of the warhead and decoy is the premise and foundation of the target recognition. 
Middle segment target characteristic

Table 1 Ballistic missile flight parameters

\begin{tabular}{|c|c|c|c|}
\hline Range/km & $\begin{array}{c}\text { Maximum flight } \\
\text { altitude/km }\end{array}$ & $\begin{array}{c}\text { Maximum flying } \\
\text { speed/ }(\mathrm{km} / \mathrm{s})\end{array}$ & $\begin{array}{c}\text { Mid flight } \\
\text { time/min }\end{array}$ \\
\hline 3500 & 687 & 4.9 & 14.2 \\
\hline 3000 & 595 & 4.6 & 12.7 \\
\hline 2500 & 486 & 4.2 & 11.1 \\
\hline 2000 & 338 & 3.6 & 8.9 \\
\hline 1500 & 259 & 3.2 & 7.3 \\
\hline 1000 & 180 & 2.7 & 5.8 \\
\hline
\end{tabular}

Be known by Table 1(the selected point trajectory of the table is $\theta=45^{\circ}$ ), flying in the middle of a ballistic missile is out of the atmosphere, flight speed is about $3 \mathrm{~km} / \mathrm{s}$,flight time is around 10 min.Compared with the boost phase and terminal,there are obvious characteristics in the middle segment flight of the ballistic missile,so ballistic missile segment is the defense key area.The main features of the middle segment flight of the ballistic missile :

(1)High altitude.Outside the atmosphere,the missile is difficult to carry out effective,relatively fixed trajectory.Therefore,for the missile interceptor has low requirement .

(2)Target group will no longer be a single.In order to increase the penetration of ballistic missiles in the middle segment,it will be throwing out some jamming equipment,such as projectile fragments, bait, warhead, etc.This is also the main reason why the target recognition

(3)The middle segment belong to the "face" defense.The middle segment is a stage of the longest flight time of a ballistic missile, about $80 \%$ to $90 \%$ of the whole trajectory,so it has a large defense area.Compared to the "terminal point" for defense,the middle segment belong to the "face" defense,can save a lot of troops.

\section{Middle segment motion characteristics}

In the course of the middle flight, there is a difference between the target and the decoy, which is mainly reflected in the:

(1) Warhead attitude control. In order to maintain the stability of the warhead during reentry into the atmosphere, it can improve the accuracy of the warhead. Warhead in the middle segment of the orbit to enter attitude control.There are many ways of attitude control, the spin is the most commonly used in the middle of the warhead attitude control.

(2) Bait random roll motion. For bait and other targets, generally do not do the attitude control,will present the roll and other random movement.In the course of the bait release, it is inevitable to produce lateral disturbance to the warhead,The spin axis of the projectile type targets will rotate around the axis of a directional symmetry axis,is the precession.

\section{Analysis of infrared recognition technology for middle segment target of ballistic missile}

Through the research and analysis of the middle segment of the target group,it is found that the following main infrared characteristic parameters can be used to identify the true and false warhead effectively by the correlation between the quality of the target and the shape of the object:temperature, radiation intensity,infrared imaging.

\section{Temperature and rate of change recognition}

When the target group enters the middle segment flight, it is exposed to the extremely low temperature outer space environment and the entry into the atmosphere at the beginning of the reentry segment.Changes of target temperature reflects the heat absorption rate of $\mathrm{a}_{\mathrm{v}}$ and $\varepsilon_{\text {IR }}$ radiation rate information,the warhead weight target heat absorption rate and emissivity ratio of $\mathrm{a}_{\mathrm{v}} / \varepsilon_{\mathrm{IR}}$, namely the heat capacity,change faster than light $\operatorname{target}^{[2]}$. Can be based on this phenomenon,to target the heat capacity changes to distinguish the relative weight of the target, and then analysis is the warhead class target or other decoy.Therefore, through the measurement of target infrared detector short wave and medium wave of the $\mathrm{a}_{\mathrm{v}} / \varepsilon_{\mathrm{IR}}$ can effectively identify the target weight. 
After entering the middle of a period of ballistic missiles, the temperature of the warhead quickly reduced, and then tend to stabilize the equilibrium temperature.The infrared decoy can be heated and other measures to raise the temperature, so it is difficult to identify target by the temperature alone.However, there is a certain relationship between the temperature change rate and the quality of the target. Different targets have different values in flight,and there are differences in the law of change:Due to the use of the attitude control and other heat sources, coupled with the greatest quality of the warhead, so in the same external environment, the temperature change of the warhead is much slower than the bait ${ }^{[3]}$,also the infrared signal is stronger than the bait.Based on this principle,long wave infrared two-color array can be composed of a radiation ratio thermometer, by ratio of radiation power spectrum measurement target in two infrared emissivity, to eliminate the influence caused by the different physical properties of the target surface, to determine the relative temperature of each object in the target group, record the change process ,then deduced the relative mass of each object ${ }^{[4]}$.

The measurement accuracy of temperature depends on the ratio of radiation power, such as infrared decoy system field meet target occlusion, or target distance changes, as long as their impact on the two band close to the same, it has no significant effect on the measurement results.Oak Ridge National Laboratory has developed a cantilever structure of the infrared detector,Can detect the temperature of the order of less than $10^{-6}$,this shows that a target can be detected only $1 / 10000$ higher than its ambient temperature ${ }^{[5]}$. With such a high sensitivity of the infrared detector, any bullet will have no place to hide.

\section{Infrared image recognition technology}

Through the infrared sensor signal processing to target imaging, can get the target of the gray characteristics and changes, analysis a series of infrared image, you can get a target of the state of motion.Figure 1 shows the flow chart of target recognition using infrared imaging.

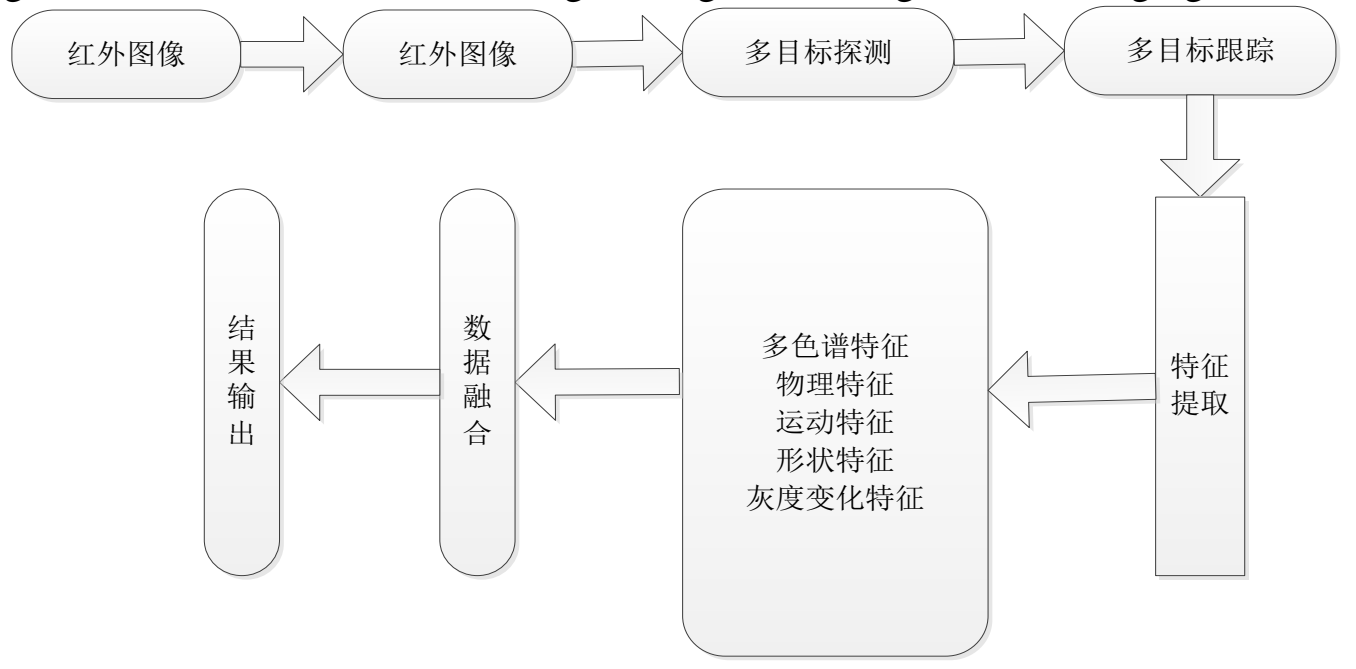

Fg1 Schematic diagram of infrared synthetic recognition

With the space based infrared system (SBIRS) application in large scale pixel focal plane array staring infrared imaging hardware and ultra high speed image processing software technology, the infrared imaging technology of target recognition is more and more important ${ }^{[6]}$. At present, in the field of infrared image recognition, the use of gray scale and motion characteristics is the most widely used in the field of infrared image recognition.

(1) Gray characteristic

Gray refers to the use of black tone to represent the object, that is, with the black as the reference color, different saturation of the black to display the image. Each gray image has a luminance value from $0 \%$ (white) to $100 \%$ (black). Infrared imaging can be used to analyze the infrared image of many targets in flight, during the middle flight, the gray level of the warhead is relatively flat, and the bait changes with time.Infrared imaging can not only identify the real warhead from numerous flying objects, but also can analyze the target and determine its thin vulnerable position,achieve effective interception. From the United States in December 5, 2008 Alaska California Kodiak 
launch a comprehensive long-range ballistic missile target ${ }^{[7]}$,over the Pacific Ocean 200km,the interceptor successfully intercepted the target at the speed of $10 \mathrm{~km} / \mathrm{s}$.But the test target could not successfully release for enemy missile penetration simulation device "bait".In spite of this, the director of the Bureau of missile defense still believes that the test is successful.Because the target warhead and booster rockets in final stage after the separation, the last stage rocket is still associated with a bullet flying, but rely on the ground and space-based infrared sensor interceptor system and its infrared sensor infrared imaging or separate the last stage rocket (the equivalent of simple bait) and warhead, and successfully intercepted.

(2) Movement characteristics

The motion characteristics can be through a series of infrared images get.The change of relative velocity after the separation of the projectile in the middle of the middle of the initial stage due to the difference in the quality of the separation of the momentum conservation of momentum and the separation of momentum.Middle segment movement,the warhead due to shape rules, the center of gravity is stable, its movement is relatively stable and the fastest, the bait is in motion in the presence of tumbling. Select a small field of view $\left(1^{\circ}---3^{\circ}\right)$,gaze tracking, increasing the integration time, according to the target infrared motion image sequences, you can identify the true and false warhead.

\section{Infrared radiation characteristic recognition technology}

The radiation intensity is the radiation flux transmitted by the point source in the solid angle of the unit in a certain direction,Units as w/sr.The radiation intensity of the target is determined by the surface temperature and emissivity of the target,includes two parts: the background radiation of the radiation and the reflection of its own emission,warhead in the middle of the main background radiation is the direct solar radiation, the earth's reflection of the solar radiation and the earth's radiation.The influencing factors include the quality of the target, the surface coating material, the thickness of the shell and so on.

Because of the different target with different surface materials, to eliminate the effects of emission rate caused by different physical properties of the target surface ${ }^{[8]}$, infrared array can be used to form a double color than the radiation thermometer, the ratio of radiation power spectrum measurement target in two infrared, to determine the relative temperature the level of each object in the group, recorded the change process, and then derive the relative quality of the size of each object, through the analysis of the quality of the size of the complete identification.Table 2 shows the variation range of the characteristic parameters of various infrared targets in the middle segment.

Table 2 Various infrared target characteristic parameter variation range

\begin{tabular}{|c|c|c|c|c|c|}
\hline infrared target & $\begin{array}{c}\text { initial } \\
\text { temperature } \mathrm{T}_{0}\end{array}$ & $\begin{array}{c}\text { Temperature } \\
\text { change rate } \\
\mathrm{dT} / \mathrm{dt}\end{array}$ & $\begin{array}{l}\text { Radiatio } \\
\text { n rate } \\
\varepsilon \lambda \text { Chang } \\
\text { e times }\end{array}$ & $\begin{array}{c}\text { Projected area } \\
\mathrm{A}_{\mathrm{p}} \text { Change } \\
\text { times }\end{array}$ & $\begin{array}{l}\text { Radiation } \\
\text { intensity } \\
\text { change rate } \\
\text { dp/dt Range } \\
\text { of change }\end{array}$ \\
\hline Missile debris & about $600 \mathrm{k}$ & fast & 1.8 & $1 \sim 125$ & larger \\
\hline Warhead & $\begin{array}{c}\text { After } \\
\text { aerodynamic } \\
\text { heating } \\
\text { about700k }\end{array}$ & slow & 1 & 1 & Very small \\
\hline Balloon bait & $\begin{array}{c}\text { room } \\
\text { temperature } \\
(300 \mathrm{k})\end{array}$ & fast & 1 & 1 & small \\
\hline
\end{tabular}

From the table 2, it can be seen that there are two kinds of infrared targets with different features in the middle of flight:one kind is the radiation intensity change is not big, but their surface temperature difference is bigger,such as warheads and balloons; the other is that the initial value of the surface temperature is close to the initial value, but the difference of their radiation intensity is 
relatively large,such as missiles and warheads.So,as long as the infrared detector to measure the infrared target group of the various surface temperature and radiation intensity change rate,it is confirmed that the surface temperature of the target group is high,and infrared target with small change of radiation intensity (heat capacity) is the incoming warhead.

\section{Future development trend}

Target recognition technology is one of the core technologies in the ballistic missile defense system, which largely determines the success or failure of the anti missile system. Infrared technology for the identification of the middle and reentry trajectory of incoming ballistic flight is considered as an effective means of identification.

At present, space-based infrared system in the United States has been put in use, its anti missile system of infrared detection, tracking and recognition system has reached the world advanced level,application of the latest achievements in the field of many infrared optical technology,the principle of technical scheme is feasible ${ }^{[9]}$.However, it still has some shortcomings, the most serious problem is that it is not fast and effective to identify the real warhead in the complex threat target group.For example, in order to cooperate with the development of STSS task,the ground-based midcourse defense system (GMDS, Ground-Based Midcourse Defense System), from 1997 to now publicly perform 17 interception test ${ }^{[10]}$. One of the four integrated flight test (IFT-4,5,8,9) in the use of a small amount of simple bait (1 3 ball bait),only IFT-8 intercept success;other 20 non intercept tests, IFT-1A, 2, and 2B, CMCM-2A used a complex decoy,the number reached 10, at this time the identification is very difficult.

Therefore, the practicability and reliability of infrared recognition still need to be further improved. Combined with the reality of our country, this paper think that in the field of infrared detection and recognition, our country should give priority to the development of the following three aspects:

(1)Hardware development.The study with coverage of short wave infrared to long wave infrared band has a high sensitivity, high uniformity, large size, with a dual color or multi color capability of the anti radiation focal plane device,improve the complex electromagnetic background reliability.

(2)Experimental simulation verification.Live-fire target practice costly,scientifically and reasonably simulated the infrared characteristics and the variation law of warhead and decoy under various optical background conditions, and in various stages of flight establishment of the corresponding dynamic identification and verification technology and system standards will be the focus of the study.

(3)Establishment ground radar net and infrared detector information fusion system.At present and in the future for some time, infrared photoelectric recognition technology is not accurate,stable to identify spatially complex, uncertain target groups.Under the background of network in the information age, information processing as the center,appropriate selection of fusion techniques rational use of sensors to complement and verify information,in order to improve the recognition effect of complex target group will be a focus on the development of the field.

\section{Reference}

[1]LU Wei-ning,LI Ke-da,HE Li-ping,et al.Ballistic missile attack and Defense Technology[M].China Aerospace Publishing House,2007

[2]LI qun-zhang. Research on infrared optical recognition methods of ballistic missiles seeker during middle course and re -entry phase [J]. Infrared and Laser Engineering.2009,28(5):1-5.

[ 3 ]CHEN Bo-liang.Important applications of IRFPA imagingdevices[J].Infrared and Laser Engineering ,2010,34 (2):168-182. 
[4]ZHANG Jian, MAO Er-ke, ZHAO Bao-jun, et al. Method for measuring the similarity between infrared images of target and its decoy [J]. Opto-Electronic Engineering, 2007, 34(9): 5-9.

[5]WU Xia, ZHOU Yan, YANG Long-po, et al. Missile recognition based on the fuzzy dynamic AHP algorithm [J]. Opto-Electronic Engineering, 2009, 36(9):6-11.

[6]CHEN Fu-qiang, LIN Ya-feng. Opto-electrical technology application in the informatization warfare [J]. Infrared and Laser Engineering,2008,37(2):366-370.

[7] Missile Defense Agency Ballistic Missile Defense System (BMDS)[R]. 2007,www.mda.mil/

[8]TIDROW M Z.MDA IR sensor technology program and applications,[C],Proceeding Of SPIE,Infrared Technology and Application XXIX,2005,5047:39 43

[9]CHEN Ping-ping, XU Qing, JIN You-lin. Analyze for infrared antagonize during the ballistic missile middle flying course [C]// The 18th Opto -Electronic Technology and Academy Communion Conference Discourses Collection,2014,10:131-135.

[10]YANG Wei, ZHANG Jian-qi, LIU Jin-song. Theory computation of infrared radicalization characteristic of flying warhead [J].Infrared and Laser Engineering,2005,34(1):42-45. 